\title{
CALIDAD DEL EMPLEO EN LA ZONA SUR AUSTRAL DE CHILE*
}

Ariel $\operatorname{Arcos}^{\mathrm{a}}$

Luz Maria Ferrada

*DOI: https://doi.org/10.18601/01245996.v21n41.09. Recepción: 06-062018, modificación final: 01-04-2019, aceptación: 14-05-2019. Sugerencia de citación: Arcos, A. y Ferrada, L. M. (2019). Calidad del empleo en la zona sur Austral de Chile. Revista de Economía Institucional, 21(41), 213-234.

${ }^{a}$ Estudiante del doctorado en Economía, [ariel.arcos@alumnos.ucn.cl], [https://orcid.org/0000-0002-4926-5021].

b Doctora en Economía Aplicada y Gestión Regional. Docente e Investigadora, Universidad de Los Lagos de Chile, Osorno, Chile, [lferrada@ulagos.c1], [https://orcid.org/0000-0002-0481-7670]. 


\section{Calidad del empleo en la zona Sur Austral de Chile}

Resumen Este artículo analiza las condiciones laborales de tres regiones del sur austral de Chile -Los Lagos, Aysén y Magallanes- entre 1990 y 2011, usando indicadores sintéticos que incluyen cuatro dimensiones: ingresos, seguridad social, jornada y contrato laboral. Se encuentra que las condiciones son significativamente diferentes -Magallanes tiene la mejor posición relativa y Los Lagos la menor-, que los indicadores no mejoraron sustancialmente con respecto a 1990, solo en estas dos hubo mejoras significativas al final del periodo.

Palabras clave: condiciones laborales, empleo, calidad del empleo; JEL: J08, R23

\section{Job quality in Southern-Austral regions of Chile}

Abstract The working conditions of three regions of the southern south of Chile are analyzed: Los Lagos, Aysén and Magallanes, constructing synthetic indicators by region in the long term, through 4 dimensions: income, social security, working day and contract. It is obtained that the conditions are significantly different between regions. Magallanes has the best relative position and Los Lagos least; In addition, the indicators have not improved substantially, compared to the initial period, only in the last two there is a significant improvement.

Keywords: Labor conditions, employment, employment quality; JEL: J08, R23

\section{Qualidade do emprego no sul do Chile}

Resumo Este artigo analisa as condições de trabalho de três regiões do sul do Chile -Los Lagos, Aysén e Magallanes- entre 1990 e 2011, utilizando indicadores sintéticos que incluem quatro dimensões: renda, seguridade social, jornada de trabalho e contrato. Verifica-se que as condições são significativamente diferentes -Magallanes tem a melhor posição relativa e Los Lagos, a menor- e que os indicadores não melhoraram substancialmente em relação a 1990, apenas nessas duas regiões houve melhorias significativas no final do período.

Palavras-chaves: condições de trabalho, emprego, qualidade do emprego; JEL: J08, R23 
Dara entender la situación del mercado laboral se suele recurrir P a medidas como tasas de participación laboral, desempleo y ocupación, que son insuficientes para evaluar la calidad del empleo, como también señalan la OIT y la Ocde. En este artículo se calculan indicadores de las condiciones de empleo en las regiones de la zona sur austral de Chile para estimar su calidad y hacer comparaciones en el largo plazo.

La zona sur austral, conocida como Patagonia Chilena, está formada por tres regiones adyacentes: Los Lagos ${ }^{1}$, Aysén y Magallanes. Es un amplio territorio con muchas fricciones espaciales, baja densidad de población y distante de los centros económicos del país. Allí se producen bienes transables, provenientes de la extracción de recursos naturales: productos silvo-agropecuarios y pesca en el norte, y de minería en el sur. La pesca y la minería son muy dinámicas en el país, muestran un fuerte crecimiento desde los años noventa y absorben un alto número de empleos no calificados.

Un estudio de todas las regiones chilenas (Cáceres y Zúñiga, 2013) señala que en el periodo 1998-2006 no aumentó el índice de calidad del empleo. En las regiones que se estudian aquí, ellos encuentran indicadores de calidad del empleo y dinámicas muy diferentes. Magallanes tuvo un mejoramiento significativo, Los Lagos una situación estable y Aysén una posición intermedia. Y, además, que la disponibilidad de trabajadores mejora la calidad del empleo; ese efecto es mayor en Los Lagos y Aysén. Esta es la base de las dos hipótesis de trabajo que se prueban en este artículo: 1) en el largo plazo, la calidad del empleo ha aumentado en las tres regiones; y 2) hay factores diferentes, entre años y regiones, que explican la calidad del empleo.

El artículo evalúa la calidad del empleo en la zona mencionada e identifica su variación en el largo plazo. Para ello se construye un indicador de calidad del empleo con datos que permitan hacer comparaciones. Primero, se estima un indicador sintético de las condiciones de empleo en las tres regiones; luego, se determina si es estadísticamente diferente entre regiones; después se analiza la estabilidad de los resultados entre 1990 y los demás periodos; por último, se evalúa el impacto de las variables demográficas.

Los trabajos sobre calidad del empleo no han examinado en detalle esta zona. Un estudio focalizado en este territorio puede ayudar

${ }^{1}$ Desde 2007 la estructura político-administrativa pasó de 13 a 15 regiones, dividiendo la región de Los Lagos. Para mantener la consistencia y por representatividad estadística de los datos, se utiliza la estructura anterior: Los Lagos incluye la actual región de Los Ríos. 
a proponer políticas diferenciadas más efectivas. La primera sección del artículo muestra los antecedentes de los enfoques sobre la calidad del empleo en América Latina y los avances de Chile en esta materia; la segunda describe la metodología y los datos utilizados, y la tercera, sintetiza los resultados. Por último, se exponen las conclusiones.

\section{ANTECEDENTES}

En esta sección se resumen los enfoques utilizados para construir medidas de calidad del empleo, así como la evidencia de América Latina y de Chile, para fundamentar la elección del método que se describe más adelante.

\section{ENFOQUES TEÓRICOS}

Un enfoque muy utilizado es partir de la utilidad del empleo para los trabajadores, además del salario, e incluye la jornada de trabajo, las tareas que desempeñan y los aspectos subjetivos que cada trabajador revela sobre las características de su cargo. Así, se han propuesto medidas que consideran aspectos objetivos y subjetivos (Somarriba et al., 2010).

Otro enfoque hace énfasis en los aspectos objetivos: la calidad del empleo es mejor en la medida en que satisface las necesidades de la persona y está ligado al enfoque de capacidades de Sen: un empleo de buena calidad es aquel que permite que el trabajador sea y haga lo que valora, de modo que logra sus objetivos personales.

En América Latina se suelen utilizar otros dos enfoques: los mercados segmentados y el trabajo decente. La idea central del primero es que hay una dicotomía entre el mercado primario, con empleos de alta calidad, y el mercado secundario, con empleos de baja calidad (Piore, 1968; Doeringer y Piore, 1971, Williamson et al., 1975 y Taubman y Wachter, 1986). El segundo establece ciertas condiciones para definir un empleo de calidad: "condiciones de libertad, equidad, seguridad y dignidad humana [...] los derechos de los trabajadores son protegidos, y cuentan con remuneración adecuada y con protección social"(OIT, 1999 ; p. 4). En cambio, en un trabajo precario hay inseguridad del empleo, bajos salarios, no hay protección social y existen obstáculos para la sindicalización (OIT, 2012). En este caso la calidad del empleo se estudia a través de las condiciones laborales.

En suma, no hay manera única de abordar y medir la calidad del empleo, por su carácter multidimensional, y se han propuesto diversas maneras de combinar las distintas dimensiones. Entre los métodos 
para construir indicadores sintéticos, en América Latina es bastante conocido el utilizado por Farné (2003).

\section{EVIDENCIA PARA AMÉRICA LATINA}

De acuerdo con Farné (2003), en Colombia hay sectores con empleos de alta calidad: administración pública, servicios sociales, sector energético y financiero. Posso (2010) coincide en que se concentran en los dos últimos sectores mencionados; además, señala que un mayor nivel educativo y empresas de gran tamaño favorecen el empleo de buena calidad.

En Ecuador, Messier y Floro (2008) estudian la calidad del empleo según la formalidad, y encuentran que el empleo formal es de mejor calidad que el informal. Aunque los mayores ingresos del sector formal no son sinónimo de mejor calidad, pues una jornada laboral adecuada y beneficios no salariales como el seguro de salud son más valorados por los trabajadores.

Marull (2010) estima un indicador sintético de calidad del empleo en Ecuador y Bolivia, y encuentra que los trabajadores independientes tienen empleos de mejor calidad. En cambio, Martínez (2012) encuentra que, en Venezuela, los trabajadores independientes tienen empleos de baja calidad.

Roethlisberger y Weller (2011) indican que, en el conjunto de los países latinoamericanos, la calidad del empleo es sensible al ciclo económico. En el periodo 1996-2002, de bajo crecimiento económico, el empleo fue de baja calidad. En 2002-2007, de mayor crecimiento, mejoró la calidad; pero se mantuvo una brecha en desmedro de las mujeres y los trabajadores rurales.

Farné et al. (2011) muestran que el crecimiento económico de Colombia de 2002 a 2010 se reflejó en un aumento de la calidad del empleo. En Chile, Infante y Sunkel (2004) también encontraron una correlación positiva entre el crecimiento económico y la calidad del empleo entre 1990 y 2000.

\section{Calidad del EMPleo en Chile}

De acuerdo con Sehnbruch (2004), en Chile el empleo de alta calidad tiene una correlación positiva con las siguientes variables: género masculino, nivel educativo, edad, tamaño de empresa y sector de servicios. Por su parte, Sehnbruch y Ruiz (2010), con datos de 2000, 2003 y 2006 , concluyen que el $60 \%$ de los trabajadores labora en condiciones muy deficientes, y que el nivel educativo, la experiencia laboral, el tipo 
de contrato y la situación de dependencia son los aspectos que más influyen en la calidad del empleo.

Mc-Clure (2010) muestra que los quintiles de menor calidad del empleo están formados en su mayoría por mujeres. Alarcón y Santos (2008) argumentan que la segregación por sexo y la subvaloración del trabajo femenino están asociadas al tamaño de las empresas, la categoría ocupacional y la rama de actividad. Huneeus et al. (2012), que estiman la probabilidad de tener un empleo de mala calidad, no encuentran evidencia de heterogeneidad entre género, sector público y privado, pero sí entre tamaño de empresas y sindicalización.

En su estudio por regiones, Cáceres y Zúñiga (2013) estiman la calidad del empleo usando el método propuesto por Chacón (1999) y Farné (2003). Así obtienen un indicador sintético para 13 regiones en los años 1998, 2000, 2003 y 2006. Los resultados de nuestras tres regiones indican que hay diferencias significativas, salvo en Magallanes y Los Lagos en 1998, cuya situación era muy desventajosa frente a la del país, aunque al final del periodo hubo un cambio notable. En 2006 Magallanes se ubicó en el primer lugar mientras que en 1998 estaba en el penúltimo. Los Lagos se ha mantenido en una baja posición, solo subió dos posiciones y en 2006 ocupó el octavo lugar. La posición de Aysén es intermedia. Solo en 1998 tuvo una posición ventajosa en la macro zona, pero inferior a la media del país. Aunque en 2006 se ubicó entre las cuatro regiones con mejor calidad del empleo. Estos autores encuentran que, en las tres regiones, la educación es una de las variables con mayor efecto, el cual es mucho mayor en Los Lagos y Aysén; además, que el grupo con más desventaja es el de trabajadores de mayor edad. Esto coincide con los resultados de Ferrada y Ferrada (2018), quienes encuentran que los trabajadores de más de 65 años tienen los empleos de menor calidad.

Por último, Ferrada (2017) encuentra que los aspectos subjetivos tienen efectos significativos en la satisfacción con el empleo y que vivir fuera de la región metropolitana tiene un efecto positivo significativo.

\section{DATOS Y MÉTODO}

\section{ÁREA DE ESTUdio}

En este apartado se describen algunas características relevantes de cada región. En Los Lagos vivía el 7,1\% de la población del país en 2011; el 32\% pertenecía a pueblos originarios, y el 70\% era urbana. Los sectores que demandaban más empleo era: silvo-comercio y silvo-agropecuario, restaurantes y hoteles, manufactura, pesca y 
construcción (Casen, 2011), y su participación en el PIB nacional era del 3,7\% (Banco Central, 2011). En Aysén solo vivía el 0,6\% de la población del país, un $23 \%$ perteneciente a pueblos originarios, y un $85 \%$ urbana. La mitad del empleo regional era proporcionada por el comercio, los restaurantes y hoteles, la administración pública, el sector agropecuario y la construcción (Casen, 2011). Su aporte al PIB nacional era del 0,5\%, y las actividades más importantes eran la pesca y la construcción (Banco Central,2011). En Magallanes vivía el 0,9\% de la población total, un $23 \%$ perteneciente a pueblos originarios y un $97 \%$ residente en zonas urbanas. Los principales sectores demandantes de empleo eran: comercio, restaurantes y hoteles, construcción, transporte y administración pública, que en conjunto absorbía el 51\% (Casen, 2011). Su aporte al PIB nacional era del 0,9\%, y se destacaban la minería y la manufactura (Banco Central, 2011).

En suma, en las tres regiones, que cubren el $41 \%$ del territorio, habita un $9 \%$ de la población del país. De modo que una de sus particularidades es la baja densidad de población. Magallanes y Aysén reciben subsidios estatales para compensar la lejanía geográfica.

\section{FUENTES DE INFORMACIÓN}

La información que se utiliza proviene de las diez bases de datos de la Encuesta Nacional de Caracterización Socioeconómica de Chile (Casen) de 1990 a 2011. Cabe señalar que la encuesta Casen de 2013 en adelante utiliza un nuevo método para estimar el ingreso, y no es posible la comparar los resultados con los años anteriores; por ello, las estimaciones se realizan hasta 2011.

Las observaciones corresponden a trabajadores hombres de 15 a 65 años de edad, y mujeres de 15 a 60 años ${ }^{2}$, que dicen ser asalariados o empleados del sector público y que responden todas las preguntas relacionadas con las variables de interés. Se excluyen las categorías definidas en la encuesta como: "patrón o empleador", "trabajador por cuenta propia" y "familiar no remunerado".

\section{DIMENSIONES Y VARIABLES}

Las dimensiones y variables utilizadas se eligieron con base en el trabajo de Farné (2003) y los de Mc-Clure (2008), Alarcón y Santos (2008) y Cáceres y Zúñiga (2013) sobre Chile. E1 indicador sintético incluye las siguientes dimensiones o componentes: ingresos (W),

${ }^{2}$ La edad de hombres y mujeres corresponde al rango entre su ingreso a la población en edad de trabajar y el año de jubilación. 
jornada laboral (JL), contrato laboral (CL) y seguridad social (SS). La selección de las variables fue determinada por la disponibilidad de información en las bases de datos utilizadas; se describen en el cuadro 1 y se explican a continuación.

Cuadro 1

Descripción de variables por dimensión

\begin{tabular}{llcc}
\hline \multicolumn{1}{c}{ Dimensión } & \multicolumn{1}{c}{ Variable } & Efecto & Tipo \\
\hline Ingresos (W) & Logaritmo natural del ingreso por hora del empleo & $(+)$ & Numérica \\
& ocupación principal, a pesos de 2011 & & \\
& Salario mayor a la línea de la pobreza & $(+)$ & Dicotómica \\
& Salario mayor al mínimo legal de cada año & $(+)$ & Dicotómica \\
\hline Jornada laboral & Trabaja en más de un empleo & $(-)$ & Dicotómica \\
$(\mathrm{JL})$ & Jornada laboral completa, según a la ley & $(+)$ & Dicotómica \\
& Jornada laboral inferior a completa, según la ley & $(-)$ & Dicotómica \\
& Jornada laboral superior a completa, según la ley & $(-)$ & Dicotómica \\
\hline Contrato laboral Tiene contrato de trabajo escrito & $(+)$ & Dicotómica \\
$(\mathrm{CL})$ & Tiene contrato de trabajo indefinido & $(+)$ & Dicotómica \\
\hline Seguridad social Cotiza para seguro de salud & $(+)$ & Dicotómica \\
$(\mathrm{SS})$ & Cotiza para seguro de jubilación & $(+)$ & Dicotómica \\
\hline
\end{tabular}

Fuente: elaboración propia.

Para la dimensión $\mathrm{W}$ se toma el logaritmo natural del ingreso por hora de trabajo del empleo principal, como proponen Alarcón y Santos (2008) y Cáceres y Zúñiga (2013). Además, se incluyen dos variables que, conforme al enfoque de trabajo decente, forman parte de esta dimensión, una de ellas identifica si se tienen ingresos superiores a la línea de pobreza, y la otra si el salario es mayor que el mínimo legal.

Con base en Farné (2003) la dimensión JL incluye el número de empleos y variables relacionadas con la jornada laboral, según la legislación chilena de cada año. Se supone que tener trabajo y jornada completa favorece la calidad del empleo, mientras que una jornada laboral menor no la favorece e indica subempleo. Los trabajadores con más de un empleo y/o jornada mayor que la legal están en condición de sobreempleo, sacrifican horas de ocio y así se reduce su bienestar; por ello su efecto negativo en la dimensión JL (signo en el cuadro 1).

Además de salario apropiado y trabajo de tiempo completo es necesario saber si hay contrato formal, lo que da protección legal, y en lo posible que sea a término indefinido pues da mayor estabilidad laboral. Así, en la dimensión CL se incorporan las variables "existencia de contrato"y "modalidad de contratación”, ambas con efecto positivo pues son características de un empleo deseable. Por último, un empleo con protección social -seguro de pensión, enfermedad y salud- proporciona mayor bienestar a los trabajadores. Ambas variables tienen un efecto positivo. 
A diferencia de Farné (2003), en las dimensiones JL, SS y CL se incluye un mayor número de variables, pero en forma dicotómica, para captar más información de cada dimensión.

\section{ESTIMACIÓN DE UN INDICADOR DE CALIDAD DEL EMPLEO (ICE)}

Roethlisberger y Weller (2012) señalan que no hay un criterio objetivo para elegir las dimensiones y las variables ni para ponderarlas en un indicador sintético. Y Verdera (2001, p. 11) indica que "un indicador compuesto que agregue otros indicadores siempre es arbitrario por la necesidad de darles pesos”. Por ello, aquí se construye un ICE que evite la subjetividad en la elección de las ponderaciones, y sean determinadas por el comportamiento de los datos.

Para determinar el valor de cada dimensión se estandarizan las variables con el método de re-escalamiento min-máx ${ }^{3}$, para que estén en las mismas unidades de medida y sean comparables. Las variables con efectos positivos en la calidad del empleo se estandarizan así:

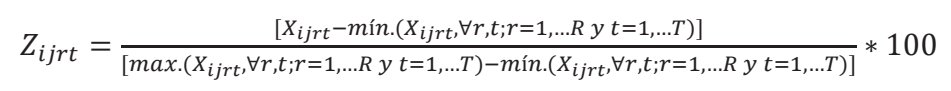

Así, las variables con efectos negativos corresponden a $\left[1-Z_{i j r t}\right] . Z_{i j r t}$ es el valor de la variable estandarizada $j$, para el individuo $i$ en el año $t$ $(\mathrm{T}=10)$ en la región $r(\mathrm{R}=3)$, cuyo valor se sitúa entre 0 y 100 , de mala a buena calidad. Después de estandarizar las variables se estiman las ponderaciones en cada dimensión, según la proporción de la desviación estándar de cada variable en cada base de datos:

$$
P_{j t}=S D Z_{j t} / \sum_{j=1}^{N} S D Z_{i j t}
$$

donde $P_{j t}$ es la ponderación de la variable $j$ en el año $t, S D Z_{j t}$ la desviación estándar de la variable estandarizada $j$ en el año $t$ y $\sum_{j=1}^{N} S D Z_{d j r t}$ la sumatoria de las desviaciones estándar de las variables estandarizadas $j$ que componen la dimensión $d$. E1 ICE de cada dimensión se estima mediante la sumatoria ponderada de las variables que lo componen: $I C E F_{\text {dirt }}=\sum_{j=1}^{N} Z_{i j r t} * P_{j t}$

donde, ICEF $F_{\text {dirt }}$ es el valor de la dimensión $d$ para el trabajador $i$ que pertenece a la región $r$ en el año $t$.

Hasta ahora se ha expuesto el método para determinar cada dimensión del indicador sintético. Para sintetizarlo en un solo indicador (ICE) primero se estima la ponderación de cada dimensión, y luego

${ }^{3}$ Para más detalles sobre este método, ver Schuschny y Soto (2009) y Bas (2014).

Revista de Economía Institucional, vol. 2 i, N. ${ }^{\circ}$ 4I, segundo semestre/2oi9, Pp. 2 I3-234 ISSN OI 24-5996/E-ISSN 2346-2450 
se agregan. Cada dimensión entra en el ICE según la ponderación $\left(P_{d}\right)$ de la ecuación 4.

$$
P_{d}=\left(\sum_{d}^{T} S D I C E F_{d t} / \sum_{f}^{N} \sum_{d}^{T} S D I C E F_{d t}\right)
$$

Así, la ponderación de cada dimensión es el cociente entre sumatoria de la desviación estándar de la dimensión $d$ en los $t$ años y la sumatoria de la desviación estándar de las cuatro dimensiones. Esta ponderación evita la subjetividad en la asignación de los pesos de cada dimensión, y es constante en el tiempo, lo que facilita la comparación del ICE en el periodo. Por último, el ICE se calcula como la suma de cada una de las cuatro dimensiones, ponderadas así:

$I C E_{\text {irt }}=P_{W} * W+P_{C L} * C L+P_{S S} * S S+P_{J L} * J L$

\section{DIFERENCIAS EN EL ICE ENTRE REGIONES}

Para verificar si hay diferencias significativas en el ICE entre regiones, se aplica una prueba de igualdad de medias, la prueba de Levene porque no precisa homoscedasticidad. Esta se contrasta con el estadístico F de Welch (1947) - We-, por su utilidad para comparar grupos de distinto tamaño muestral, cuyas varianzas son significativamente diferentes pues en la fórmula para calcularlo (6), se incluye una ponderación basada en el tamaño de la muestra y la varianza de cada grupo (Jan y Shieh, 2014):

$W e=\left\{\frac{\sum w_{r t}\left[\bar{x}_{r t}-\left(\sum w_{r t} \bar{x}_{r t} / \sum w_{r t}\right)\right]^{2}}{(k-1)}\right\} /\left\{1+\left[\frac{2(k-2)}{k^{2}-1}\right] \sum \frac{\left[\left(1-w_{r t}\right) / \sum w_{r t}\right]^{2}}{\left(n_{r t}-1\right)}\right\}$

donde el subíndice $r$ es la región, $t$ el año de observación, $n$ y $s^{2}$, el tamaño de las muestras y la varianza por región, $\bar{x}_{r}$ la media del ICE en la región, y w es la proporción entre el tamaño de la muestra de la región $r$ en el año $t$ y la varianza de la región $r$ en $t,\left(n_{r t} / s_{r t}^{2}\right)$, y $k$ el número de regiones que se comparan.

El estadístico We así calculado sigue una distribución $\mathrm{F}$ con $(k-1)$ grados de libertad en el numerador y $\left\{\left(k^{2}-1\right) / 3 \sum\left[\left(\left(1-w_{r t}\right) / \sum w_{r t}\right)^{2} /\left(n_{r t}-1\right)\right]\right\}$ grados de libertad en el denominador.

\section{EFECTOS EN EL ICE}

Para evaluar el efecto de las variables demográficas, el capital humano, el lugar de residencia y el sector ocupacional- en el ICE se estimó un modelo cuyas variables explicativas se describen en el cuadro 2.

ICE, la variable dependiente, es el índice de calidad del empleo de cada persona de la muestra, y se especifica así: 
$I C E_{i}=\beta X_{i}+\alpha Y_{i}+\delta Z_{i}+\rho O_{i}+u_{i}$

donde $X, Y, Z$ y, $O$ son las matrices de las variables mencionadas e $i$ es la observación. Se estimaron 10 regresiones, una por periodo.

Cuadro 2

Variables explicativas del ICEG

\begin{tabular}{|c|c|c|}
\hline Tipo & Nombre & Descripción \\
\hline Demográficas & sexo & Masculino $=1 ;$ femenino $=0$ \\
\hline \multirow[t]{3}{*}{$(\mathrm{X})$} & edad1 & Tiene entre 15 y 29 años de edad=1; 0 en otros casos. \\
\hline & edad 2 & Tiene entre 30 y 44 años de edad $=1 ; 0$ en otros casos. \\
\hline & edad3 & Tiene más de 44 años de edad=1; 0 en otros casos. \\
\hline $\begin{array}{l}\text { Capital humano } \\
\text { (Y) }\end{array}$ & escolaridad & Número de años de escolaridad. \\
\hline \multirow{3}{*}{$\begin{array}{l}\text { Residencia } \\
(Z)\end{array}$} & urbano & Reside en zona urbana=1; zona rural $=0$ \\
\hline & LLagos & Reside en Los Lagos $=1 ; 0$ en los otros casos. \\
\hline & Aysén & Reside en Aysén $=1,0$ en los otros casos. \\
\hline \multirow{11}{*}{$\begin{array}{l}\text { Sector } \\
\text { o tamaño de la em- } \\
\text { presa en que trabaja } \\
\text { (O) }\end{array}$} & público & Administración pública y defensa $=1 ; 0$ para otros. \\
\hline & instrucción & Instrucción pública = 1; 0 para otros sectores. \\
\hline & doméstico & Servicio doméstico $=1 ; 0$ para otros sectores. \\
\hline & comercio & Comercio al por menor $=1 ; 0$ en otros sectores. \\
\hline & rest_hoteles & Restaurantes y hoteles $=1 ; 0$ otros sectores. \\
\hline & agropecuario & Producción agropecuaria $=1 ; 0$ otros sectores. \\
\hline & pesca & Pesca $=1 ; 0$ para otros sectores. \\
\hline & silvícola & Silvicultura $=1 ; 0$ para otros sectores. \\
\hline & empresa5 & Empresas con 5 o menos trabajadores $=1 ; 0$ otros casos. \\
\hline & empresas 49 & Empresas 6 a 49 trabajadores $=1 ; 0$ otros casos. \\
\hline & empresa50 & Empresas con 50 o más trabajadores $=1 ; 0$ otros casos. \\
\hline
\end{tabular}

Fuente: elaboración propia.

Inicialmente se usaron mínimos cuadrados ordinarios ( $\mathrm{MCO}$ ). Pero la escolaridad puede ser endógena, dado que una parte de los ingresos es explicada por el nivel educativo, y como el ingreso es parte del ICE, la escolaridad quedaría incorporada a través de la dimensión W. Si la escolaridad se considerara exógena y no lo es, se obtendrían estimaciones inconsistentes y, por tanto, resultados sesgados. Por ello se utilizaron 2 etapas, usando como instrumentos la edad y la edad al cuadrado (Mora y Ulloa, 2011).

\section{ESTABILIDAD DEL ICE}

Para comprobar si hay estabilidad en la calidad del empleo de cada región en el tiempo se usa el método propuesto por Cáceres y Zúñiga (2013), que en este caso consiste en unir todas las bases de datos en un solo archivo y luego estimar las regresiones de las variables explicativas binarias del año de cada encuesta. De este modo, el modelo se especifica así:

$I C E_{\text {ir }}=\beta_{90}+\beta_{92} \delta_{92}+\beta_{94} \delta_{94} \ldots+\beta_{11} \delta_{11}+u_{i}$ 
donde ICE es el indicador global estimado de cada trabajador $i$ perteneciente a la región $r$ en los años 1990,1992, 1994, 1996, 1998,2000, $2003,2006,2009$ y 2011 . $\beta_{90}$, el intercepto, representa el promedio del ICEG en el año base (1990), $\beta_{92}$ representa la diferencia promedio del ICEG entre 1992 y el año base, $\beta_{94}$ la diferencia promedio del ICEG entre 1994 y el año base, y así sucesivamente hasta el año 2011. Así, $\delta$ representa la variable binaria que identifica los años 1992, 1994, 1996, 1998, 2000, 2003, 2006, 1009 y 2011.

Se contrasta la hipótesis de que los coeficientes son estadísticamente nulos para probar la estabilidad del ICE entre 1990 y el año que representa cada coeficiente.

\section{RESULTADOS}

En esta sección primero se presentan los resultados del indicador global y de los indicadores por dimensión, para cada región y año; luego se verifica si hay diferencias significativas en el indicador global de empleo entre regiones y años. Después se prueba si ese resultado es estable y por último se analizan las variables que afectan el resultado del ICE en cada año. Con base en ello se prueban las hipótesis de trabajo mencionadas al comienzo.

\section{INDICADOR SINTÉTICO DE CALIDAD DEL EMPLEO EN LAS REGIONES}

Gráfica 1

ICE por región, 1990-2011

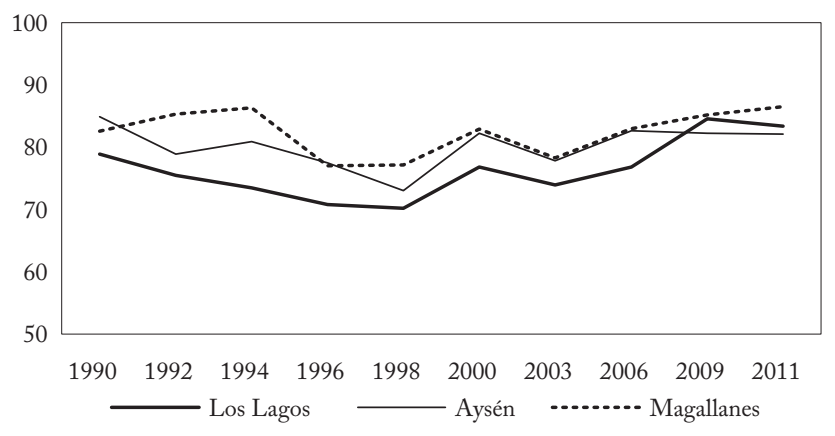

Fuente: elaboración propia.

La gráfica 1 muestra los resultados del ICE por región. En el cálculo se pondera cada dimensión según su desviación estándar (ecuación 4). La dimensión que más contribuye al índice es CL, con un promedio del 31\%, le sigue SS con el 29\%, JL con el 23\%, y por último W con 
el 17\%. Estos resultados son distintos de los de otros trabajos, en los que el ingreso es el que tiene mayor incidencia.

En general, el ICE de Los Lagos es el más bajo en todo el periodo, lo que concuerda con los resultados de Cáceres y Zúñiga (2013); pero en 2009 y 2011 iguala y supera al de Aysén. Cabe destacar que los ICE de finales del periodo, no son sustancialmente mayores que los de comienzos de los años noventa.

La gráfica 2a muestra los resultados de cada dimensión. La baja calidad del empleo en Los Lagos se debe a los bajos salarios y a menores condiciones relativas en la seguridad social y los contratos. Aunque en 2009 y 2011 hubo mejoras en la dimensión CL que se reflejaron en el índice global. En Magallanes los empleos son de mejor calidad, quizá debido a la mayor participación de los sectores de rápido crecimiento (minería) y al papel del Estado, cuyos efectos se reflejan en su mejor situación en las dimensiones SS, CL y W. Estos resultados también coinciden con los de Cáceres y Zúñiga (2013).

En la dimensión JL (gráfica 2b), se observa un comportamiento cíclico a lo largo del periodo, principalmente en Aysén, debido a las mayores variaciones de los niveles de empleo con jornada laboral superior a la completa, en comparación con las otras dos regiones.

\section{Gráfica 2}

Calidad del empleo por dimensión en cada región
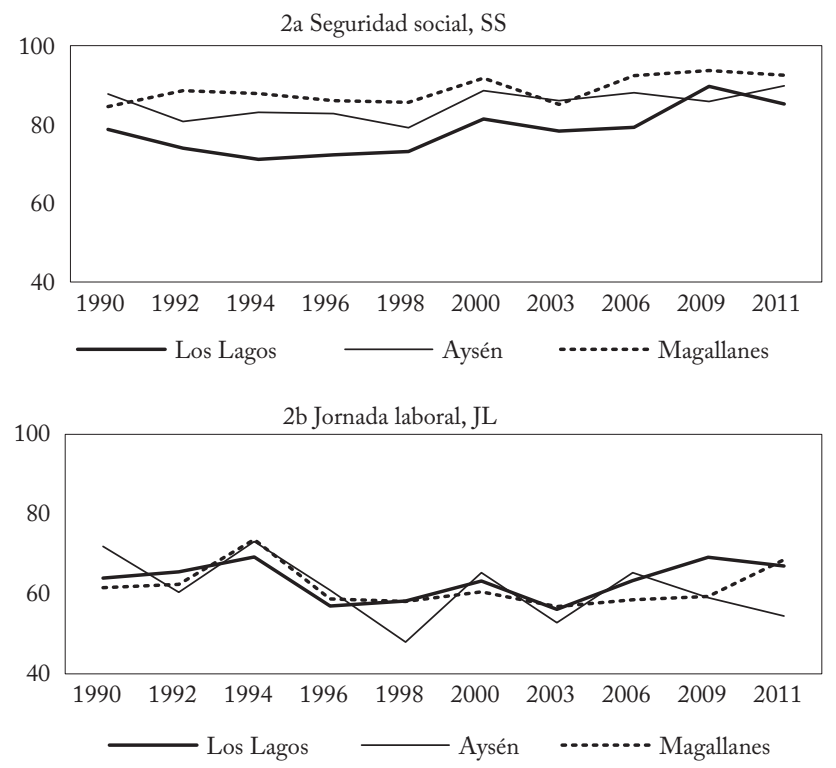

Revista de Economía Institucional, vol. 2 i, N. ${ }^{\circ}$ 4I, segundo semestre/2oi9, Pp. 2 I3-234 ISSN OI 24-5996/E-ISSN 2346-2450 
Gráfica 2 (continuación)

Calidad del empleo por dimensión en cada región
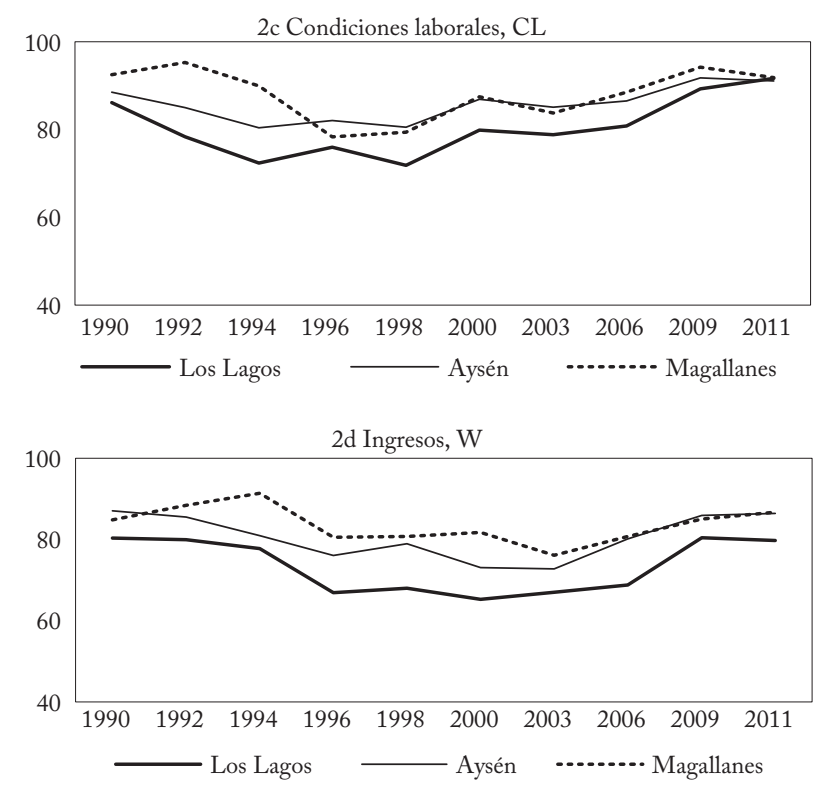

Fuente: Anexo 2, con base en encuestas Casen 1990-2011.

Desde 2003, Los Lagos y Magallanes han mejorado en la dimensión JL; mientras que en Aysén ha tendido a desmejorar desde comienzos del periodo, debido principalmente, a la reducción de los empleos con jornada laboral completa, que pasaron del 57\% en 1990 al 26\% en 2011.

En la dimensión CL (gráfica 2c), las mayores diferencias entre regiones se presentan en 1992 y 1994, cuando el índice se reduce en Aysén y Los Lagos debido a la reducción de los empleos con contrato por escrito. Desde 1998, hay similitudes en los valores y la evolución de los índices de Aysén y Magallanes, mientras que el de Los Lagos es más bajo, aunque se acerca a ellos en 2011, debido a mejoras en los empleos con contrato y con contrato indefinido. La alta ponderación de esta dimensión influye en el descenso inicial del ICE.

Aunque los salarios reales han aumentado, el índice W no ha mejorado (gráfica 2d), se redujo entre 1994 y 1996 por la disminución de los empleos con salarios mensuales superiores al mínimo legal. Aunque en Magallanes se observa una mejora entre 1990 y 1994 debido a la creciente proporción de trabajadores con salarios superiores a la línea de pobreza y al mínimo legal. En Los Lagos, el nivel relativo de las tres variables que componen la dimensión es más bajo que en 
Aysén y Magallanes, porque en estas regiones hay mayores subsidios al empleo privado y a los salarios del sector público; como ya se dijo para compensar el mayor costo de vida de habitar en esa zona distante.

\section{DIFERENCIAS DEL ICE ENTRE REGIONES}

Para determinar si hay diferencias significativas en las condiciones laborales entre las tres regiones se hizo la prueba de Welch para la igualdad de medias, cuyos resultados se muestran en el cuadro 3.

Cuadro 3

Resultados de la prueba de Welch (We)

\begin{tabular}{lrrrrrrrrrr}
\hline Año & 1990 & 1992 & 1994 & 1996 & 1998 & 2000 & 2003 & 2006 & 2009 & 2011 \\
\hline We & $1.151,7$ & $5.337,8$ & $6.913,0$ & $1.779,2$ & $1.554,4$ & $2.098,5$ & 789,8 & 2320,9 & 150,0 & 727,2 \\
\hline Prob. & 0,000 & 0,000 & 0,000 & 0,000 & 0,000 & 0,000 & 0,000 & 0,000 & 0,000 & 0,000 \\
\hline
\end{tabular}

Se encontró que, en promedio, hay diferencias estadísticamente significativas entre los ICE de Los Lagos, Aysén y Magallanes, en los diez años de estudio. Este indicador específico -que solo considera la variabilidad de la información de esas tres regiones- corrobora los resultados de Cáceres y Zúñiga (2013) para el periodo 1990-2011.

\section{ESTABILIDAD DEL ICE EN EL TIEMPO}

Por el momento, los resultados indican que la calidad del empleo es diferente en las tres regiones: la de Magallanes en primer lugar y la de Los Lagos en el último. Ahora se debe verificar si hay diferencias significativas entre el indicador de cada año y el del año inicial. Para ello se hace la prueba de estabilidad de los parámetros mencionada en la sección metodológica. El cuadro 4 muestra los resultados.

Aunque, en la mayoría de los años la calidad del empleo ha disminuido con respecto al año base, en Magallanes se observan mejoras importantes desde 2006, y en Los Lagos solo en los dos últimos años; Aysén tiene un comportamiento opuesto.

Los coeficientes son en general negativos y significativos y, por tanto, el índice tiende a ser menor que el de 1990, debido principalmente a que si bien los salarios han aumentado, su ponderación en el ICE es baja; y a que si bien las variables han mostrado mejoras, ingresan en cada dimensión según las condiciones de cada año, es decir, los criterios para construir el índice varían, de modo que se reduce la proporción de trabajadores que los cumplen. Así, por ejemplo, el salario mínimo legal pasó de 82.827 pesos mensuales en 1990 a 182.000 en 2011, lo 
que eleva el nivel de exigencia de esta variable, es decir, el número de trabajadores con un salario mayor al mínimo legal.

Cuadro 4

Prueba de estabilidad de los coeficientes

\begin{tabular}{lllllll}
\hline & \multicolumn{2}{c}{ Los Lagos } & \multicolumn{2}{c}{ Aysén } & \multicolumn{2}{c}{ Magallanes } \\
& Coef. & Err. st. rob. & Coef. & Err. st. rob. & Coef. & Err. st. rob. \\
\hline 1992 & $-3,399$ & $0,831^{* * * * *}$ & $-5,969$ & $1327^{* * * *}$ & 2,779 & $0,932^{* * * *}$ \\
1994 & $-5,389$ & $0,894^{* * * * *}$ & $-3,975$ & $1430^{* * * *}$ & 3,778 & $1135^{* * * *}$ \\
1996 & $-8,114$ & $0,958^{* * * *}$ & $-7,414$ & $1359^{* * * *}$ & $-5,527$ & $1619^{* * * *}$ \\
1998 & $-8,694$ & $0,959^{* * * *}$ & -1186 & $1304^{* * *}$ & $-5,396$ & $1575^{* * *}$ \\
2000 & $-2,100$ & $0,873^{* * * *}$ & $-2,640$ & $1322^{* * *}$ & 0,334 & 1,329 \\
2003 & $-4,938$ & $0,798^{* * * *}$ & $-7,100$ & $1479^{* * * *}$ & $-4,255$ & $1702^{* *}$ \\
2006 & $-2,077$ & $0,760^{* * * *}$ & $-2,237$ & 1,460 & 0,401 & 1,276 \\
2009 & 5,671 & $0,770^{* * * *}$ & $-2,666$ & 1,680 & 2,643 & 1,935 \\
2011 & 4,474 & $0,722^{* * * *}$ & $-2,808$ & $1026^{* * *}$ & 3,965 & $1254^{* * *}$ \\
Const. & 78,889 & $0,537^{* * * *}$ & 84,890 & $0,863^{* * * *}$ & 82,540 & $0,738^{* * * *}$ \\
Observaciones & & 32.939 & & 7.994 & & 7.042 \\
Prob > F & & 0 & & 0 & & 0 \\
$\mathrm{R}^{2}$ & 0,0342 & & 0,0238 & & 0,0338 \\
ECM & & 24,507 & & 21,222 & & 18,893 \\
\hline
\end{tabular}

${ }^{*} \mathrm{p}<0,1 ; * * \mathrm{*}<0,05, * * * \mathrm{p}<0,01$.

Fuente: elaboración propia.

\section{EFECTO DE LAS VARIABLES EN EL ICE POR AÑO}

Para determinar si hay factores diferentes entre años y regiones que explican el resultado del ICE, se hicieron estimaciones para cada año, considerando como variables de referencia la edad (de 30 a 44 años), si el trabajador es mujer y si reside en áreas rurales. Y en Magallanes, si trabaja en empresas de 6 a 49 trabajadores, y en el sector privado distinto de servicio doméstico, comercio, restaurantes y hoteles, agropecuario, silvícola y pesca. Los resultados se muestran en el cuadro 5.

En casi todos los años, la calidad del empleo asalariado disminuye para los trabajadores jóvenes (15 a 29 años) con respecto a los de mediana edad (de 30 a 44 años), y mejora notoriamente para los trabajadores de mayor edad (45 y más años). Estos resultados concuerdan con la teoría del capital humano, según la cual la edad es una buena proxy de la experiencia. No obstante, en los últimos años este resultado pierde significancia estadística. Así mismo, igual que en otros trabajos se encuentra que la educación tiene un efecto positivo sobre la calidad del empleo en la mayoría de los años, pero en 2009 y 2011 ese efecto no es estadísticamente significativo. Vale la pena 
mencionar que durante todo el periodo aumentó el número promedio de años de escolaridad, de 9,3 en 1990 a 11,3 en 2011.

Cuadro 5

Impacto de las variables sobre el ICE, regresión en 2 etapas

\begin{tabular}{|c|c|c|c|c|c|c|c|c|c|c|}
\hline & 1990 & 1992 & 1994 & 1996 & 1998 & 2000 & 2003 & 2006 & 2009 & 2011 \\
\hline escolaridad & $\begin{array}{l}2,50^{\circ *} \\
(1,244)\end{array}$ & $\begin{array}{l}2,04^{*} \\
(1,118)\end{array}$ & $\begin{array}{l}-3,47^{\prime \prime} \\
(1,711)\end{array}$ & $\begin{array}{l}6,06 \\
(1,570)\end{array}$ & $\begin{array}{l}1,83^{\circ} \\
(1,098)\end{array}$ & $\begin{array}{l}2,54^{*} \\
(1,485)\end{array}$ & $\begin{array}{l}2,33^{\circ} \\
(0,924)\end{array}$ & $\begin{array}{l}1,65^{\circ} \\
(0,690)\end{array}$ & $\begin{array}{l}1,74 \\
(1,476)\end{array}$ & $\begin{array}{l}0,76 \\
(0,723)\end{array}$ \\
\hline sexo & $\begin{array}{l}1,95 \\
(1,823)\end{array}$ & $\begin{array}{l}2,73 \\
(1,936)\end{array}$ & $\begin{array}{l}-6,95^{\circ} \\
(2,614)\end{array}$ & $\begin{array}{l}1,89 \\
(2,707)\end{array}$ & $\begin{array}{l}-0,69 \\
(1,686)\end{array}$ & $\begin{array}{l}1,43 \\
(1,850)\end{array}$ & $\begin{array}{l}2,31^{\circ} \\
(1,402)\end{array}$ & $\begin{array}{l}0,37 \\
(1,041)\end{array}$ & $\begin{array}{l}3,43 \\
(2,118)\end{array}$ & $\begin{array}{l}2,24^{+3} \\
(0,829)\end{array}$ \\
\hline edad & $\begin{array}{l}-4,88^{\cdots} \\
(1,182)\end{array}$ & $\begin{array}{l}0,31 \\
(1,225)\end{array}$ & $\begin{array}{l}-0,83 \\
(1,813)\end{array}$ & $\begin{array}{l}-10,05 \\
(1,712)\end{array}$ & $\begin{array}{l}-6,20^{-1} \\
(1,518)\end{array}$ & $\begin{array}{l}-4,17^{\prime \prime} \\
(1,628)\end{array}$ & $\begin{array}{l}-7,57^{\prime} \\
(1,254)\end{array}$ & $\begin{array}{l}-3,73^{-1} \\
(1,224)\end{array}$ & $\begin{array}{l}-3,13 \\
(1,238)\end{array}$ & $\begin{array}{l}-1,72^{* \prime} \\
(0,863)\end{array}$ \\
\hline edad3 & $\begin{array}{l}6,21^{* \prime} \\
(2,823)\end{array}$ & $\begin{array}{l}2,49 \\
(3,024)\end{array}$ & $\begin{array}{l}-7,31^{-1} \\
(4,131)\end{array}$ & $\begin{array}{l}13,33 \\
(4,640)\end{array}$ & $\begin{array}{l}5,44^{-*} \\
(2,308)\end{array}$ & $\begin{array}{l}4,12 \\
(2,835)\end{array}$ & $\begin{array}{l}4,42^{--\prime} \\
(1,709)\end{array}$ & $\begin{array}{l}5,56 \\
(1,258)\end{array}$ & $\begin{array}{l}2,19 \\
(1,897)\end{array}$ & $\begin{array}{l}1,49 \\
(1,216)\end{array}$ \\
\hline urbano & $\begin{array}{l}-0,76 \\
(2,219)\end{array}$ & $\begin{array}{l}1,71 \\
(2,710)\end{array}$ & $\begin{array}{l}6,44^{\circ *} \\
(3,050)\end{array}$ & $\begin{array}{c}-10,84 \\
(4,664)\end{array}$ & $\begin{array}{l}0,53 \\
(2,385)\end{array}$ & $\begin{array}{l}-4,92^{* \prime} \\
(2,709)\end{array}$ & $\begin{array}{l}-6,33^{+\cdots} \\
(1,578)\end{array}$ & $\begin{array}{l}-4,26 \\
(1,351)\end{array}$ & $\begin{array}{l}-2,23 \\
(2,116)\end{array}$ & $\begin{array}{l}-0,99 \\
(0,944)\end{array}$ \\
\hline LLagos & $\begin{array}{l}-0,03 \\
(1,053)\end{array}$ & $\begin{array}{l}-7,82^{*-1} \\
(0,982)\end{array}$ & $\begin{array}{l}-11,07^{\prime} \\
(2,013)\end{array}$ & $\begin{array}{l}-3,23^{\prime \prime} \\
(1,927)\end{array}$ & $\begin{array}{l}-3,07^{\prime \prime} \\
(1,472)\end{array}$ & $\begin{array}{l}-1,79 \\
(1,601)\end{array}$ & $\begin{array}{l}-3,88^{\prime \prime} \\
(1,602)\end{array}$ & $\begin{array}{l}-4,81^{\prime \prime} \\
(1,325)\end{array}$ & $\begin{array}{l}1,21 \\
(1,970)\end{array}$ & $\begin{array}{l}-3,15 \\
(0,974)\end{array}$ \\
\hline Aysén & $\begin{array}{l}5,66^{-\infty} \\
(1,458)\end{array}$ & $\begin{array}{l}-4,17^{*} \\
(1,546)\end{array}$ & $\begin{array}{l}-3,74 \\
(2,026)\end{array}$ & $\begin{array}{l}4,58^{*} \\
(2,398)\end{array}$ & $\begin{array}{l}-0,69 \\
(1,888)\end{array}$ & $\begin{array}{l}3,84^{*} \\
(2,075)\end{array}$ & $\begin{array}{l}0,05 \\
(2,135)\end{array}$ & $\begin{array}{l}0,68 \\
(1,636)\end{array}$ & $\begin{array}{l}-0,15 \\
(2,623)\end{array}$ & $\begin{array}{l}-3,17^{-1} \\
(1,024)\end{array}$ \\
\hline público & $\begin{array}{l}-7,61^{-\prime} \\
(2,418)\end{array}$ & $\begin{array}{l}0,13 \\
(2,770)\end{array}$ & $\begin{array}{l}1,35 \\
(5,181)\end{array}$ & $\begin{array}{l}-10,70^{\prime \prime} \\
(4,428)\end{array}$ & $\begin{array}{l}-4,47^{\circ} \\
(2,588)\end{array}$ & $\begin{array}{l}-1,12 \\
(2,055)\end{array}$ & $\begin{array}{l}-5,20^{+\prime} \\
(2,528)\end{array}$ & $\begin{array}{l}-6,37^{*-1} \\
(1,756)\end{array}$ & $\begin{array}{l}-4,73 \\
(1,455)\end{array}$ & $\begin{array}{l}-8,25^{-\infty} \\
(1,296)\end{array}$ \\
\hline instrucción & $\begin{array}{l}-10,18 \\
(5,213)\end{array}$ & $\begin{array}{l}-7,38 \\
(4,665)\end{array}$ & $\begin{array}{l}15,16^{*} \\
(6,975)\end{array}$ & $\begin{array}{l}-19,31 \\
(5,657)\end{array}$ & $\begin{array}{l}-1,94 \\
(3,225)\end{array}$ & $\begin{array}{l}-3,60 \\
(4,843)\end{array}$ & $\begin{array}{l}-5,31 \\
(3,541)\end{array}$ & $\begin{array}{l}-5,08^{\circ} \\
(2,979)\end{array}$ & $\begin{array}{l}-2,49 \\
(2,868)\end{array}$ & $\begin{array}{l}-0,47 \\
(2,291)\end{array}$ \\
\hline doméstico & $\begin{array}{l}-1,56 \\
(5,065)\end{array}$ & $\begin{array}{l}7,83^{*} \\
(4,624)\end{array}$ & $\begin{array}{l}-24,94 \\
(6,068)\end{array}$ & $\begin{array}{l}-3,39 \\
(5,926)\end{array}$ & $\begin{array}{l}-11,05^{\prime \prime} \\
(4,201)\end{array}$ & $\begin{array}{l}-11,45^{\prime \prime} \\
(5,406)\end{array}$ & $\begin{array}{l}-2,54 \\
(3,633)\end{array}$ & $\begin{array}{l}-13,16 \\
(2,793)\end{array}$ & $\begin{array}{l}-8,50 \\
(5,249)\end{array}$ & $\begin{array}{l}-12,05 \\
(2,983)\end{array}$ \\
\hline comercio & $\begin{array}{l}2,24 \\
(1,712)\end{array}$ & $\begin{array}{l}1,03 \\
(1,978)\end{array}$ & $\begin{array}{l}6,93^{*+} \\
(2,188)\end{array}$ & $\begin{array}{l}-2,21 \\
(2,517)\end{array}$ & $\begin{array}{l}3,27^{\circ} \\
(1,793)\end{array}$ & $\begin{array}{l}3,85^{\circ} \\
(1,789)\end{array}$ & $\begin{array}{l}4,24^{\circ} \\
(1,589)\end{array}$ & $\begin{array}{l}0,48 \\
(1,733)\end{array}$ & $\begin{array}{l}4,41 \\
(1,416)\end{array}$ & $\begin{array}{l}2,55 \\
(0,894)\end{array}$ \\
\hline rest_hoteles & $\begin{array}{l}-3,50 \\
(3,248)\end{array}$ & $\begin{array}{l}4,66 \\
(3,161)\end{array}$ & $\begin{array}{l}2,40 \\
(3,308)\end{array}$ & $\begin{array}{l}3,64 \\
(4,490)\end{array}$ & $\begin{array}{l}0,44 \\
(3,444)\end{array}$ & $\begin{array}{l}5,69 \\
(4,004)\end{array}$ & $\begin{array}{l}6,59^{-m} \\
(2,312)\end{array}$ & $\begin{array}{l}-0,36 \\
(2,712)\end{array}$ & $\begin{array}{l}-1,68 \\
(2,873)\end{array}$ & $\begin{array}{l}-3,43 \\
(3,725)\end{array}$ \\
\hline pesca & $\begin{array}{l}-0,85 \\
(2,601)\end{array}$ & $\begin{array}{l}2,86 \\
(2,217)\end{array}$ & $\begin{array}{l}-10,88^{\prime \prime} \\
(4,347)\end{array}$ & $\begin{array}{l}-4,25 \\
(4,658)\end{array}$ & $\begin{array}{l}-7,79^{\circ} \\
(4,199)\end{array}$ & $\begin{array}{l}4,03 \\
(4,100)\end{array}$ & $\begin{array}{l}3,32^{\circ} \\
(1,770)\end{array}$ & $\begin{array}{l}0,90 \\
(1,768)\end{array}$ & $\begin{array}{l}0,05 \\
(3,074)\end{array}$ & $\begin{array}{l}2,81 \\
(2,144)\end{array}$ \\
\hline agropecuario & $\begin{array}{l}0,05 \\
(3,006)\end{array}$ & $\begin{array}{l}2,89 \\
(2,807)\end{array}$ & $\begin{array}{l}-11,34 \\
(4,126)\end{array}$ & $\begin{array}{l}1,03 \\
(3,243)\end{array}$ & $\begin{array}{l}-1,10 \\
(3,541)\end{array}$ & $\begin{array}{l}8,82 \\
(4,367)\end{array}$ & $\begin{array}{l}2,41 \\
(2,349)\end{array}$ & $\begin{array}{l}2,36 \\
(1,992)\end{array}$ & $\begin{array}{l}2,08 \\
(2,370)\end{array}$ & $\begin{array}{l}2,49 \\
(1,698)\end{array}$ \\
\hline silvícola & $\begin{array}{l}-0,94 \\
(4,108)\end{array}$ & $\begin{array}{l}-0,52 \\
(3,458)\end{array}$ & $\begin{array}{l}-12,11 \\
(4,775)\end{array}$ & $\begin{array}{l}-8,91^{*} \\
(4,625)\end{array}$ & $\begin{array}{l}-12,76^{\prime \prime} \\
(5,298)\end{array}$ & $\begin{array}{l}-5,56 \\
(3,422)\end{array}$ & $\begin{array}{l}-1,86 \\
(2,544)\end{array}$ & $\begin{array}{l}-7,69= \\
(2,820)\end{array}$ & $\begin{array}{l}-3,78 \\
(3,747)\end{array}$ & $\begin{array}{l}-1,09 \\
(1,697)\end{array}$ \\
\hline empresa5 & $\begin{array}{l}-8,24 \\
(1,381)\end{array}$ & $\begin{array}{l}-242,00 \\
(1,438)\end{array}$ & $\begin{array}{l}-20,64 \\
(2,080)\end{array}$ & $\begin{array}{l}-5,32 \\
(2,439)\end{array}$ & $\begin{array}{l}-12,28 \\
(2,160)\end{array}$ & $\begin{array}{l}-11,94 \\
(2,046)\end{array}$ & $\begin{array}{l}-15,99 \\
(1,780)\end{array}$ & $\begin{array}{l}-13,34 \\
(1,595)\end{array}$ & $\begin{array}{l}-9,19 \cdots \\
(2,122)\end{array}$ & $\begin{array}{l}-9,48^{-1-} \\
(1,519)\end{array}$ \\
\hline empresa50 & $\begin{array}{l}4,93^{\cdots-} \\
(1,017)\end{array}$ & $\begin{array}{l}1,22 \\
(1,433)\end{array}$ & $\begin{array}{l}4,81^{\cdots+} \\
(1,632)\end{array}$ & $\begin{array}{l}4,22 \\
(1,907)\end{array}$ & $\begin{array}{l}6,48^{\cdots} \\
(1,555)\end{array}$ & $\begin{array}{l}1,66 \\
(1,894)\end{array}$ & $\begin{array}{l}3,80^{-\cdots} \\
(1,277)\end{array}$ & $\begin{array}{l}4,00 \\
(1,002)\end{array}$ & $\begin{array}{l}2,30^{\circ} \\
(1,317)\end{array}$ & $\begin{array}{l}1,54 \\
(0,803)\end{array}$ \\
\hline _cons & $\begin{array}{l}59,56^{-1} \\
(1,276)\end{array}$ & $\begin{array}{l}60,12 \\
(10,923)\end{array}$ & $\begin{array}{l}127,49^{-\cdots} \\
(18,009)\end{array}$ & $\begin{array}{l}28,72^{*} \\
(15,305)\end{array}$ & $\begin{array}{l}60,06 \\
(11,707)\end{array}$ & $\begin{array}{l}58,68 \\
(16,089)\end{array}$ & $\begin{array}{l}60,43^{\cdots} \\
(10,176)\end{array}$ & $\begin{array}{l}70,24 \\
(7,518)\end{array}$ & $\begin{array}{l}65,62 \\
(17,582)\end{array}$ & $\begin{array}{l}78,95^{\circ+*} \\
(8,820)\end{array}$ \\
\hline Obser & 3.041 & 2.902 & 2.734 & 2.844 & 2.850 & 4.999 & 6.312 & 7.506 & 4.761 & 9.939 \\
\hline & 454,38 & 179,29 & 465,92 & 304,2 & 608,96 & 366,96 & 550,78 & 578,81 & 167,4 & 414,47 \\
\hline Prob $>$ & 0,000 & 0,000 & 0,000 & 0,000 & 0,000 & 0,000 & 0,000 & 0,000 & 0,000 & 0,000 \\
\hline Raiz del ECM & 20,684 & 24,284 & 26,821 & 28,258 & 23,255 & 22,336 & 22,54 & 21,801 & 18,556 & 16,604 \\
\hline
\end{tabular}

Entre paréntesis, errores estándar; ${ }^{*} \mathrm{p} \leq 0.1,{ }^{* *} \mathrm{p} \leq 0.05,{ }^{* * *} \mathrm{p} \leq 0.01$.

Fuente: elaboración propia.

En cuanto al género, en los tres últimos años del periodo, la calidad del empleo mejora para los hombres, y en los demás años no hay diferencias significativas entre hombres y mujeres, lo que llama la atención dada la brecha de salarios por género que encuentran otros trabajos, como el de Ferrada y Montaña (2012). Esto puede obedecer a que la dimensión de ingresos es el componente con menor ponderación en el índice global y a que solo se considera el trabajo asalariado. Quizá las mayores diferencias se encuentren en el segmento no asalariado.

Para analizar el efecto del sector económico se dividieron algunos sectores para evaluarlo con mayor precisión: Por ejemplo, el sector silvo-agropecuario se dividió en silvícola y agropecuario, lo que fue 
posible porque la muestra considera las tres regiones cada año y el tamaño resultante es aceptable.

E1 sector público y de educación tienen efectos negativos, quizá debido a la alta proporción de trabajadores sin contrato indefinido, y esta dimensión es la que tiene mayor ponderación en el índice global. $\mathrm{El}$ sector comercio tiene un efecto positivo en la mayoría de los años, mientras que el empleo en el servicio doméstico es de muy baja calidad, debido a que según la legislación chilena hasta 2010 la jornada laboral era mayor que la de los demás trabajadores y el salario mínimo era menor. Aunque desde marzo de 2011 rige una nueva normativa sobre el salario de los trabajadores domésticos, no se refleja en los resultados de ese año y el coeficiente estimado sigue siendo negativo ${ }^{4}$. El efecto de los sectores pesquero y agropecuario no es estable y pocas veces es significativo, aunque el del sector silvícola es claramente negativo.

Por su parte, la calidad del empleo aumenta en las empresas de mayor tamaño. En empresas de 50 o más trabajadores es mayor que en las de 10 a 49 trabajadores, En las empresas de 5 o menos trabajadores la calidad es mucho menor. Se constata además que el índice de calidad del empleo es menor en Los Lagos que en Magallanes, mientras que el efecto de residir en Aysén varía entre años.

En resumen, en la zona de estudio los trabajadores de más de 29 años, los que trabajan en empresas de mayor tamaño y los que desempeñan actividades comerciales tienen empleo de mejor calidad que los trabajadores jóvenes, los que trabajan en microempresas y los que se desempeñan en el sector público, la silvicultura y el servicio doméstico.

\section{CONCLUSIONES}

Para estudiar la calidad del empleo en la zona sur austral de Chile se construyó un índice que sintetiza algunos aspectos objetivos de las condiciones laborales. Según la literatura, las dimensiones elegidas constituyen una buena aproximación. En la construcción del índice (ICE) se utilizaron datos de las diez encuestas Casen con información comparable entre 1990 y 2011, para detectar las diferencias anuales entre regiones y evaluar sus cambios en el periodo.

Si bien los indicadores sintéticos son muy utilizados, es difícil estimar las ponderaciones, y ese es un aporte de este trabajo, donde se estiman, tanto para las dimensiones como para las variables con base en la variabilidad entre regiones y años, de modo que el peso de cada componente varía cada año. Este procedimiento revela la

${ }^{4}$ Desde 2015, la ley exige igualar la jornada laboral de los trabajadores de labores domésticas a la de los otros trabajadores con contrato laboral. 
contribución de cada dimensión, y permite comparar las diferencias entre las regiones de Magallanes, Aysén y Los Lagos. En todos los años las dimensiones del ICE con mayor ponderación son "contrato laboral" y "seguridad social", con un promedio del 31\% y el 29\%, respectivamente. Les sigue "jornada laboral" con un 23\% e "ingresos" con un $17 \%$.

Un resultado que difiere de los de trabajos anteriores es la menor importancia relativa de la dimensión "ingresos", cuya variabilidad puede obedecer a su composición: el salario mensual, en logaritmos naturales, y dos variables binarias (si el salario es superior a la línea de pobreza y si es mayor que el mínimo legal). Así, esta dimensión y el ICE no se ven muy afectados por la tendencia creciente de los salarios reales.

Con respecto a la primera hipótesis de trabajo de este artículo se encuentra que la calidad del empleo de 1992 a 2006 no es diferente de la de 90, solo mejora en 2009 y 2011 en Los Lagos, y en 2011 en Magallanes. Hay dimensiones que en 20 años no muestran mejoras sustantivas en estas regiones, con contrato laboral y seguridad social, que son las más importantes en la estimación del ICE. También ha habido cambios de política pública que tienen efectos en el ICE: jornada de trabajo, salario mínimo, línea de la pobreza y contrato laboral, que generaron un punto de inflexión del ICE en 1998, año que coincide con una caída de la actividad económica.

Con respecto a la segunda hipótesis se encuentra que trabajar en los sectores pesquero y silvícola tiene un efecto negativo en la calidad del empleo; aunque ese efecto es menor en los últimos años. En cambio, trabajar en comercio y educación tiene un impacto positivo, que no es significativo en 2009 y 2011. Y, en contra de lo esperado, trabajar en el sector público tiene un efecto negativo en la mayoría de los años, quizá por la importancia de la contratación por honorarios. Mientras que trabajar en empresas de gran tamaño mejora la calidad del empleo. En los últimos años han perdido significancia la escolaridad, que la persona tenga 45 o más años de edad y resida en el sector urbano.

En general, los resultados de este trabajo concuerdan con los de Cáceres y Zúñiga (2013); Magallanes tiene los mejores indicadores de calidad del empleo y Los Lagos los más bajos; también, hay diferencias significativas entre regiones en todos los años y los parámetros son inestables. Un aporte de este trabajo, que examina la situación en el largo plazo, es que solo al final del periodo mejoran las condiciones laborales, y que la dimensión "jornada laboral” es similar en las tres regiones, aunque tiende a diferenciarse al final del periodo. Además, 
algunas características de la macro zona explican el comportamiento del indicador global de calidad del empleo.

Estos resultados invitan a profundizar el estudio de las condiciones laborales en estas tres regiones, explorando métodos para superar la falta de datos comparables del periodo más reciente.

\section{REFERENCIAS BIBLIOGRÁFICAS}

Alarcón, R. y Santos, H. (2008). Una mirada longitudinal de la calidad del empleo y sus diferencias desde la perspectiva de género: Encuesta Panel Casen 1996 - 2001 - 2006. Documento de trabajo del Departamento de Estudios Mideplan.

Arraz, J. M., García-S., C. et al. (2018). Índice de calidad del empleo. Asempleo. Madrid: Universidad de Alcalá.

Bas, M. (2014). Estrategias metodológicas para la construcción de indicadores compuestos en la gestión universitaria. Tesis Doctoral, Universitat Politécnica de Valencia.

Cáceres, D. y Zúñiga S. (2013). Disparidades regionales y estabilidad de la calidad del empleo en Chile: 1998-2006. Gestión y Política Pública, 22(1), 203-243.

Chacón, B. (1999). Calidad del empleo y pobreza en chile, 1990-1996. En R. Infante (ed.), La calidad del empleo: La experiencia de los paises latino americanos y de los Estados Unidos (pp. 181-237). Santiago, Chile: OIT.

Doeringer, P. y Piore, M. (1971). Internal labor markets and manpower analysis. Cambridge, Mass.: Massachusetts.

Farné, S. (2003). Estudio sobre la calidad del empleo en Colombia. Documento de trabajo. Lima: Oficina Internacional del Trabajo-Oficina Subregional.

Farné, S. (2012). La calidad del empleo en América Latina a principios del siglo XXI. Bogotá: Universidad Externado de Colombia.

Farné, S., Vergara, C. et al. (2011). La calidad del empleo en medio de la flexibilización laboral. Colombia 2002-2010. Documento de trabajo del Observatorio del Mercado de Trabajo y la Seguridad Social. Bogotá: Universidad Externado de Colombia.

Ferrada, L. (2017). Determinantes y diferencias territoriales en materia de satisfacción laboral: el caso de Chile. Revista de la Cepal, 123, 137-157.

Ferrada, L. y Ferrada, M. (2018). Calidad del empleo de los adultos mayores en Chile, un factor de envejecimiento activo. Papeles de Población, 24(95), 43-68

Huneeus, F., Landerretche, O. et al. (2012). Multidimensional measure of job quality: Persistence and heterogeneity in a developing country. Documento de trabajo del Departamento de Economía, Universidad de Chile, Santiago de Chile.

Infante, R. y Sunkel, G. (2004). Trabajo decente y calidad de vida familiar 1990-2000. Santiago de Chile: Oficina Internacional del Trabajo. 
Jan, S. y Shieh, G. (2014). Sample size determinations for Welch's test in one-way heteroscedastic ANOVA. British Journal of Mathematical and Statistical Psychology, 67, 72-93.

Martínez, A. (2012). Calidad del empleo en el mercado laboral venezolano: un análisis para el periodo 1995-2005. Gaceta Laboral, 18(2), 173-212.

Marull, C. (2010). La calidad del empleo en América Latina. La experiencia de Bolivia y Ecuador en el periodo 2001-2007. Tesis Master of Arts Interdisziplinäre Lateinamerikastudien. Freie Universitat de Berlin.

Mc-Clure, O. (2010). Calidad de los empleos según grupos ocupacionales. Documento de trabajo del Departamento de estudios del Mideplan, División Social.

Messier, J. y Floro, M. (2008). Measuring the quality of employment in the informal sector. Washington DC: Working Papers 10.

Montaña, V. y Ferrada, L. (2016). Disparidades regionales de la bancarización en Chile. Periodo 2001-2012. Banco Central de Chile, 19(2), 1-21.

Mora, J. y Ulloa, M. (2011). Calidad del empleo en las principales ciudades colombianas y endogeneidad de la educación. Revista de Economía Institucional, 13(25), 163-177.

OIT. (1999). Trabajo Decente. Memoria del Director General a 87a reunión de la Conferencia Internacional del Trabajo, Ginebra.

OIT. (2012). Del trabajo precario al trabajo decente. Ginebra.

Piore, M. (1968). On the job training and adjustment to technological change. The Journal of Human Resources, 3(4), 435-439.

Posso, C. (2010). Calidad del empleo y segmentación laboral: un análisis para el mercado laboral colombiano 2001-2006. Desarrollo y Sociedad, 65, 191-234.

Roethlisberger, C. y Weller, J. (2011). La calidad del empleo en América Latina. Documento de trabajo de la División de Desarrollo Económico. Cepal.

Schuschny, A. y Soto, H. (2009). Guía metodológica: diseño de indicadores compuestos de desarrollo sostenible. Documento de proyecto, Cepal.

Sehnbruch, K. (2004). From the quantity to the quality of employment: An application of the capability approach to the Chilean labor market. Working paper 9, Center for Latin American Studies University of California.

Sehnbruch, K. (2012). La calidad del empleo en Chile: teoría y medición. En S. Farné (comp.), La calidad del empleo en América Latina a principios del siglo XXI (pp. 264-301). Bogotá: Universidad Externado de Colombia.

Sehnbruch, K. y Ruiz-T., J. (2010). The quality of employment: A missing link in development strategies? The example of the Chilean labour market. Presentación en el Seminario Construyendo los Pilares de 1a Política Nacional de Empleo, Santiago de Chile.

Somarriba, N., Merino, M. C. et al. (2010). La calidad del trabajo en la Unión Europea. Estudios de Economía Aplicada, 28(2), 1-22.

Taubman, P. y Watcher, M. (1986). Segmented labour markets. En: O. Ashenfelter y R. Layard (eds.), Handbook of labour economics (pp. 1183-1217). Nueva York: Elsevier. 
Verdera, F. (2001). Calidad del empleo: conceptos, medición y políticas, Taller sobre Calidad del Empleo en Colombia. Bogotá: Universidad Externado de Colombia.

Welch, B. (1947). The generalization of Student's Problem when several different population variances are involved. Biometrika, 1-2(34), 28-35.

Williamson, O., Wachter, M. et al. (1975) La relación de empleo: el análisis del intercambio idiosincrásico. En L. Toharia (ed.), El mercado de trabajo. Teoría y aplicaciones. Madrid: Alianza Editorial. 